\title{
Increased Risk of Infection and Mortality in Women after Cardiac Surgery Related to Allogeneic Blood Transfusion
}

\author{
MARY A.M. ROGERS, Ph.D., M.S., ${ }^{1,2}$ NEIL BLUMBERG, M.D., F.A.S.C.P., ${ }^{3}$ \\ JOANNA M. HEAL, M.B.B.S., M.R.C.P., ${ }^{4}$ and GEORGE L. HICKS, Jr., M.D. ${ }^{5}$
}

\begin{abstract}
Background: Infection and mortality rates are greater in women than in men after cardiac surgery. This study was conducted to assess whether allogeneic blood transfusion could partially account for this gender difference, as transfusion has been associated with immunomodulation.

Methods: A cohort study was conducted in 380 patients at the University of Rochester Medical Center. Subjects were adult patients who underwent primary coronary artery bypass graft (CABG) surgery, primary valve replacement surgery, or both. Information was collected about blood components transfused, as well as postoperative infection, pulmonary dysfunction, and in-hospital mortality.

Results: Women were more likely to receive allogeneic red blood cells (RBCs) or platelets than men (odds ratio [OR] 21.6, 95\% CI 3.8, 124.2) and a greater quantity of blood than men. Patients who received allogeneic blood were 4.4 times more likely to develop an infection than those who did not $(95 \%$ CI $1.5,13.2)$. There was a positive linear correlation between number of units of blood received and number of days with fever $(p<0.001)$ and hospital length of stay $(p<0.001)$. This was particularly evident in patients who received four or more units of nonleukoreduced blood components. Women had a greater risk of infection $(p=$ $0.005)$, pulmonary dysfunction $(p=0.005)$, and mortality $(p=0.007)$ than men during hospitalization.

Conclusions: One reason for the greater mortality in women after cardiac surgery may be the increased likelihood of receiving nonleukoreduced allogeneic RBCs and platelets. Transfusion increased the risk of infection; infection, then, increased the likelihood of pulmonary dysfunction and mortality.
\end{abstract}

\footnotetext{
${ }^{1}$ Division of General Medicine, Department of Internal Medicine, University of Michigan, Ann Arbor, Michigan.

${ }^{2}$ Patient Safety Enhancement Program, VA Medical Center and University of Michigan Health System, Ann Arbor, Michigan.

${ }^{3}$ Transfusion Medicine Unit, Department of Pathology and Laboratory Medicine, University of Rochester Medical Center, Rochester, New York.

${ }^{4}$ Hematology-Oncology Unit, Department of Medicine, and ${ }^{5}$ Cardiac Surgery Unit, Department of Surgery, University of Rochester, Rochester, New York.
} 


\section{INTRODUCTION}

$\mathbf{I}_{n}^{N}$ N A RECENT REVIEW on revascularization in women, the author stated: "Although our understanding of the gender differences in outcomes following coronary revascularization has increased, what is missing is a clear concept of how these gender paradoxes translate into increased morbidity and mortality in women." ${ }^{1}$ We report here evidence suggesting an explanation for this gender gap in morbidity and mortality after cardiac surgery.

The pathway leading to greater mortality in women may begin with the decision to administer a blood transfusion. Blood transfusion is the most common procedure during hospitalization, and its use has increased 64\% from 1997 to $2003 .{ }^{2}$ Cardiac surgery accounts for approximately 20\% of all allogeneic blood transfusions in the United States, although there is considerable variability among hospitals regarding the amount of blood components administered. ${ }^{3}$ Although transfusion may be necessary to prevent or treat tissue hypoxia, the immunomodulatory effects of allogeneic blood transfusion have been recognized for decades. ${ }^{4-6}$ Transfusion-related immunomodulation has been shown to result in an increased risk of nosocomial infection and mortality in various patient cohorts. ${ }^{7-13}$

The proposed causal pathway is of particular import to women, as the decision to prescribe a transfusion conventionally rests on threshold levels of hemoglobin or hematocrit. Normal hemoglobin and hematocrit levels are lower in women, and, thus, women are more likely than men to receive a transfusion. ${ }^{14,15}$ We studied this pathway, from female gender to transfusion to infection to mortality, in patients who underwent cardiac surgery and hypothesized that this mechanism may account for some of the increased mortality risk observed in women.

\section{MATERIALS AND METHODS}

Subjects were patients who underwent primary coronary artery bypass graft (CABG) surgery, primary valve replacement surgery, or both at the University of Rochester Medical Center in Rochester, New York, from July 1 to December 31, 1997 or from July 1 to December 31, 1998. Eligibility was restricted to adults $(\geq 18$ years of age). Operations were performed by two surgeons with long-established practices at the institution.

Information was collected from cardiothoracic division records, as well as the transfusion service, laboratory information system, and patient medical records. Abstraction of information from the cardiothoracic division was blinded to the type of transfusion received. Demographic data were compiled regarding age at the time of surgery, body mass index (BMI), sex, race (white, other), and ethnicity (Hispanic, non-Hispanic). Data were also recorded regarding urgency of surgery (elective, urgent, emergency), preoperative hematocrit, ejection fraction, and comorbidities (diabetes mellitus, renal disease, hypercholesterolemia, hypertension, coronary artery disease [CAD], family history of CAD, history of myocardial infarction [MI], congestive heart failure $[\mathrm{CHF}]$, chronic obstructive pulmonary disease [COPD], and peripheral vascular disease). Numbers of units of transfused blood components were obtained from the transfusion service, as was information regarding filtration of leukocytes (yes or no). Leukoreduction of red blood cells (RBC) was conducted by filtration prior to storage at the regional blood supplier. Leukoreduction of whole blood-derived platelet concentrates was conducted by filtration after storage and immediately prior to transfusion. Leukoreduced products decreased the leukocyte count by approximately $99.9 \%$ for both prestorage-filtered RBCs and poststorage-filtered platelets.

Outcomes included in-hospital mortality, total length of stay in the hospital (days), number of hours in the intensive care unit, diagnosis of infection (all sites combined), number of days of fever (temperature $>37^{\circ} \mathrm{C}$ ), days of antibiotic therapy, and diagnosis of pulmonary dysfunction. Pulmonary dysfunction was defined operationally as any pulmonary failure or dysfunction that delayed extubation postoperatively beyond 48 hours or required reintubation. Definition of pulmonary dysfunction in general also required that imaging studies reflect bilateral pathology, no CHF, dysfunction lasting $\geq 72$ hours, and arterial oxygen pressure $<200 \mathrm{~mm}$.

The analyses were structured to assess the relationships among factors in the proposed causal pathway, from gender to transfusion to infection to pulmonary dysfunction to mortality. Categorical data were initially examined using Pearson's chi-square test or Fisher's exact test (for data with $\leq 5$ expected observations/cell). Differences in 
means were determined using Student's $t$-test, preceded by $F$ tests for differences in variance. Exact binomial 95\% confidence intervals (CI) were calculated for proportions. Pearson product-moment correlation coefficients were used to assess the strength of the association between units of blood received and continuous outcomes. Imputation by best-subset regression was performed for demographic variables with missing values prior to fitting models; this occurred in $0.5 \%$ of the subjects. Generalized linear regression models were used to model the relation between predictors and outcomes, with Newton-Raphson maximum likelihood optimization and clustering by attending surgeon. For continuous outcomes, a Gaussian distribution was used with an identity-link function. Adjusted means were centered on the mean age (67 years) and mean BMI (28 $\mathrm{kg} / \mathrm{m}^{2}$ ). For dichotomous outcomes, a binomial distribution with a logit-link function was used. Robust (Huber/White/sandwich) estimators of variance were calculated. Alpha was set at 0.05, 2-tailed. Stata/SE 9.2 was used for the analyses.

\section{RESULTS}

There were 380 subjects, $60.5 \%$ men and $39.5 \%$ women. Women were more likely than men to be older, Hispanic, and have CHF (Table 1). Women were also more likely to receive valve repair or CABG with valve repair than men in this patient population $(p=0.003)$. Women were more evenly distributed across BMI categories than men $(p=$ 0.015), although mean BMI was similar in the two groups (mean $28.0 \mathrm{~kg} / \mathrm{m}^{2}$, standard deviation [SD] 5.9 for women; mean $27.9 \mathrm{~kg} / \mathrm{m}^{2}$, SD 4.6 for men; $p=0.9036)$. At the extremes of this distribution, there were fewer patients with low BMI of $<18.5$ ( 3 women and 1 man) than patients with BMI $>40$, indicating morbid obesity (5 women and 7 men).

Table 1. Characteristics of Patients by Gender

\begin{tabular}{|c|c|c|c|}
\hline Characteristic & $\begin{array}{c}\text { Women } \\
\mathrm{n}=150 \\
\mathrm{n}(\%)\end{array}$ & $\begin{array}{c}\text { Men } \\
\mathrm{n}=230 \\
\mathrm{n}(\%)\end{array}$ & $\mathrm{p}$ value \\
\hline \multicolumn{4}{|l|}{ Age, years } \\
\hline$<60$ & $22(14.7 \%)$ & $61(26.5 \%)$ & \\
\hline $60-69$ & $32(21.3 \%)$ & $81(35.2 \%)$ & \\
\hline $70-79$ & 69 (46.0\%) & 70 (30.4\%) & \\
\hline$\geq 80$ & 27 (18.0\%) & $18(7.8 \%)$ & $<0.01$ \\
\hline Race, white & $139(92.7 \%)$ & $218(94.8 \%)$ & 0.40 \\
\hline Ethnicity, non-Hispanic & $143(95.3 \%)$ & $227(98.7 \%)$ & 0.05 \\
\hline \multicolumn{4}{|l|}{$\mathrm{BMI}, \mathrm{kg} / \mathrm{m}^{2}$} \\
\hline$<25$ & $52(34.7 \%)$ & $57(24.8 \%)$ & \\
\hline 25-29.9 & $51(34.0 \%)$ & $112(48.7 \%)$ & \\
\hline$\geq 30$ & $47(31.3 \%)$ & $61(26.5 \%)$ & 0.01 \\
\hline \multicolumn{4}{|l|}{ Urgency of surgery } \\
\hline Elective & $60(40.3 \%)$ & $99(43.0 \%)$ & \\
\hline Urgent & $80(53.7 \%)$ & $106(46.1 \%)$ & \\
\hline Emergency & $9(6.0 \%)$ & $24(10.4 \%)$ & 0.27 \\
\hline \multicolumn{4}{|l|}{ Type of surgery } \\
\hline CABG & $86(57.3 \%)$ & $170(73.9 \%)$ & \\
\hline Valve repair & $29(19.3 \%)$ & $24(10.4 \%)$ & \\
\hline CABG and valve repair & $35(23.3 \%)$ & $36(15.7 \%)$ & $<0.01$ \\
\hline Diabetes mellitus & $56(37.3 \%)$ & $70(30.4 \%)$ & 0.16 \\
\hline Renal disease & $25(16.7 \%)$ & 37 (16.1\%) & 0.88 \\
\hline Hypercholesterolemia & $81(54.0 \%)$ & $126(54.8 \%)$ & 0.88 \\
\hline Hypertension & $111(74.0 \%)$ & $151(65.7 \%)$ & 0.09 \\
\hline History of coronary artery disease & $108(72.0 \%)$ & $171(74.3 \%)$ & 0.61 \\
\hline Family history of coronary artery disease & $78(52.0 \%)$ & $123(53.5 \%)$ & 0.78 \\
\hline History of myocardial infarction & $61(40.7 \%)$ & $111(48.3 \%)$ & 0.15 \\
\hline Ejection fraction $<40 \%$ & $112(75.2 \%)$ & $179(78.5 \%)$ & 0.45 \\
\hline Congestive heart failure & $71(47.3 \%)$ & $50(21.7 \%)$ & $<0.01$ \\
\hline Chronic obstructive pulmonary disease & $18(12.0 \%)$ & $39(17.0 \%)$ & 0.19 \\
\hline Peripheral vascular disease & $22(14.7 \%)$ & $40(17.4 \%)$ & 0.48 \\
\hline
\end{tabular}


Table 2. Association between Predictors and Outcomes in Proposed Causal Pathway

\begin{tabular}{llcrrr}
\hline Predictor & \multicolumn{1}{c}{ Outcome } & Unadjusted OR & \multicolumn{1}{c}{$95 \%$ CI } & Adjusted OR $^{\mathrm{a}}$ & \multicolumn{1}{c}{$95 \%$ CI } \\
\hline Female sex & Transfusion $^{\mathrm{b}}$ & 44.6 & $5.0,394.4$ & 34.0 & $3.8,303.7$ \\
Transfusion & Infection $_{\text {Infection }}$ & 10.7 & $3.7,30.6$ & 4.4 & $1.5,13.2$ \\
Pulmonary dysfunction & Pulmonary dysfunction & 46.2 & $9.4,227.4$ & 20.5 & $4.4,95.6$ \\
& Death & 46.3 & $37.5,57.2$ & 246.9 & $23.5,2595.6$ \\
\hline
\end{tabular}

aAdjusted for age, race, ejection fraction, surgical priority, type of surgery (CABG, valve repair or both), body mass index, diabetes, renal disease, history of coronary artery disease, history of myocardial infarction, congestive heart failure, and chronic obstructive pulmonary disease.

${ }^{\mathrm{b}}$ Transfusion of red blood cells or platelets.

\section{Gender and transfusion}

Of the 150 women, $99.3 \%(n=149)$ received allogeneic RBCs or platelets during hospitalization for cardiac surgery. This compares with $77.0 \%$ of men $(p<0.001)$. The odds that a woman would receive a transfusion were 44.6 times greater than for a man $(95 \%$ CI 5.0, 394.4) (Table 2). When adjusted for demographic and clinical factors, including BMI, the odds ratio (OR) was 34.0 (95\% CI 3.8, 303.7), and with further adjustment for preoperative hematocrit, the OR remained significant at 21.6 (95\% CI 3.8, 124.2).

Although preoperative hematocrit did affect the likelihood of receiving a blood transfusion, it had less of an effect on women than men. Ninetytwo percent of men with low preoperative hematocrit $(<41 \%)$ and $100 \%$ of women with low hematocrit $(<38 \%)$ were given at least one unit of RBCs or platelets during hospitalization. In contrast, $62 \%$ of men with normal preoperative hematocrit $(41 \%-50 \%)$ received blood, and 99\% of women with normal preoperative hematocrit $(38 \%-50 \%)$ had a transfusion $(p<0.001)$. Figure 1 shows the percentage of patients who received four or more units of RBCs during hospitalization by gender and preoperative hematocrit. In women whose preoperative hematocrit was normal, $40 \%-50 \%$ received four or more units of RBCs during the course of their hospitalization.

Women were more likely than men to receive a greater quantity of blood, regardless of the type of allogeneic blood received. The mean number of units of unfiltered blood (RBCs or platelets or both) transfused in women was 9.2 (95\% CI 7.3, 11.2) compared with 6.3 units $(95 \%$ CI $4.7,8.0)$ in men $(p=0.024)$. The mean number of units of whole blood-derived random donor platelets received by women was $16.0(95 \% \mathrm{CI} 6.9,25.0)$ compared with 6.7 units $(95 \%$ CI $5.5,7.8)$ in men $(p=$
0.021). The mean number of units of prestorage leukoreduced RBCs received by women was 4.9 $(95 \%$ CI $3.9,5.8)$ compared with 3.0 units $(95 \%$ CI $2.3,3.7)$ in men $(p=0.001)$.

\section{Gender and outcomes}

Women were more likely than men to be diagnosed with infection $(p=0.005)$ or pulmonary dysfunction $(p=0.005)$ and to die during hospitalization $(p=0.007)$ (Table 3$)$. While $6.7 \%$ of women died while in the hospital, $1.3 \%$ of men died yielding a risk ratio of 5.1 (95\% CI: 1.4, 18.3). Approximately $11 \%$ of women developed pulmonary dysfunction compared with $3.9 \%$ of men (risk ratio $=2.9,95 \%$ CI 1.3, 6.3). The mean number of hours spent in an intensive care unit was longer in women than in men (129.3 hours vs. 65.3 hours, respectively, $p=0.032$ ). The mean length of stay in the hospital was also greater in women

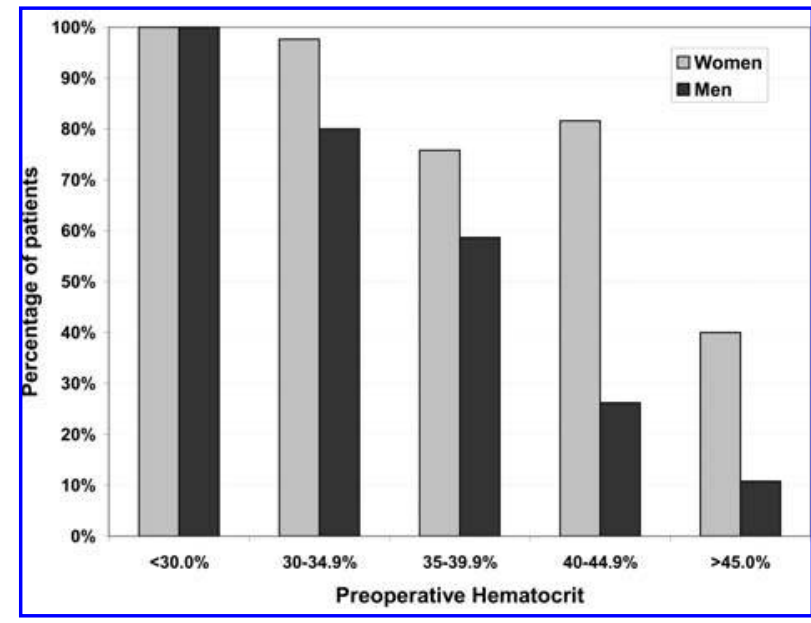

FIG. 1. Percentage of surgical patients who received four or more units of red blood cells during hospitalization, by preoperative hematocrit and gender. 
Table 3. Differences in Outcomes by Gender

\begin{tabular}{|c|c|c|c|c|c|}
\hline & \multicolumn{2}{|c|}{ Women } & \multicolumn{2}{|c|}{ Men } & \multirow[b]{2}{*}{$p$ value } \\
\hline & $\%$ or mean & $95 \% C I$ & $\%$ or mean & $95 \% \mathrm{CI}$ & \\
\hline Infection & $33.3 \%$ & $25.9,41.5$ & $20.4 \%$ & $15.4,26.2$ & $<0.01$ \\
\hline Mean days with fever & 6.6 & $5.0,8.2$ & 5.3 & $4.2,6.5$ & 0.21 \\
\hline Mean days on antibiotics & 6.0 & $3.4,8.6$ & 4.2 & $1.9,6.6$ & 0.33 \\
\hline Mean hours in intensive care & 129.3 & $70.2,188.4$ & 65.3 & $37.3,93.2$ & 0.03 \\
\hline Mean length of stay & 16.2 & $12.6,19.8$ & 10.5 & $8.9,12.1$ & $<0.01$ \\
\hline Pulmonary dysfunction & $11.3 \%$ & $6.7,17.5$ & $3.9 \%$ & $1.8,7.3$ & $<0.01$ \\
\hline In-hospital mortality & $6.7 \%$ & $3.2,11.9$ & $1.3 \%$ & $0.3,3.8$ & $<0.01$ \\
\hline
\end{tabular}

(16.2 days) compared with men (10.5 days) ( $p=$ $0.001)$.

\section{Transfusion and infection}

Patients who received RBCs or platelets were 10.7 times as likely to develop an infection during hospitalization as patients who did not receive these blood components (95\% CI 3.7, 30.6, $p=0.001$ ) (Table 2). With adjustment for demographic and clinical factors, the OR for the relation between transfusion and infection was 4.4 (95\% CI 1.5, 13.2, $p=0.009$ ).

There was a positive linear correlation between number of units of blood (RBCs or platelets or both) received and the number of days of fever during hospitalization; the correlation coefficient was $0.709(p<0.001)$. The correlation coefficient increased to $0.771(p<0.001)$ for nonleukoreduced, unfiltered blood only. Similarly, the correlation between number of units of RBCs or platelets or both transfused and number of days on antibiotic medication was significant $(r=$ $0.697, p<0.001$ ), and when only nonleukoreduced unfiltered blood was considered, the correlation coefficient was $0.758(p<0.001)$. The number of hours spent in an intensive care unit increased with the number of units of blood received ( $r=0.474, p<0.001)$, and the association was stronger when only nonleukoreduced blood was given $(r=0.726, p<0.001)$. This was similar to the pattern for hospital length of stay $(r=$ $0.494, p<0.001$ for all units of RBCs or platelets or both; $r=0.793, p<0.001$ for nonleukoreduced, unfiltered blood only).

\section{Mortality}

Of the 380 patients, 13 died while in the hospital $(3.4 \%)$. All the patients who died had re- ceived a blood transfusion. Infection was strongly associated with both mortality $(p<0.001)$ and pulmonary dysfunction $(p<0.001)$. When adjusted for demographic and clinical factors, the OR for the relation between infection and pulmonary dysfunction was 20.5 (95\% CI 4.4, 95.6, $p<0.001$ ), and the OR for the association between pulmonary dysfunction and death was 246.9 (95\% CI 23.5, 2595.6, $p<0.001$ ) (Table 2).

\section{Outcomes by type of transfusion}

Both quantity and type of transfusion (leukoreduced and nonleukoreduced) affected outcomes (Table 4). Patients who received either no units or less than four units of RBCs or platelets or both had the lowest risk of mortality. Mortality was highest $(10.5 \%)$ in patients who received four or more units of either RBCs or platelets, some of which were unfiltered. In this group, the mean number of units of unfiltered RBCs was 9.6 and of unfiltered platelets was 1.4. These patients were disproportionately women; $32 \%$ of women received this type of transfusion compared with $16.5 \%$ of men.

Compared with patients without a blood transfusion, the number of days with fever was significantly greater for those patients who received four or more units of blood components, with the exception of those who received only prestorage filtered RBCs. Patients who received four or more units of RBCs and platelets (some unfiltered) had a significantly increased number of days with fever (8.8 days) compared with patients without a transfusion (3.3 days, $p<0.001$ ) and had a significantly increased number of days with fever compared with those who received four or more units of RBCs and platelets, all of which was filtered (6.3 days, $p<0.001)$. Filtering also appeared 


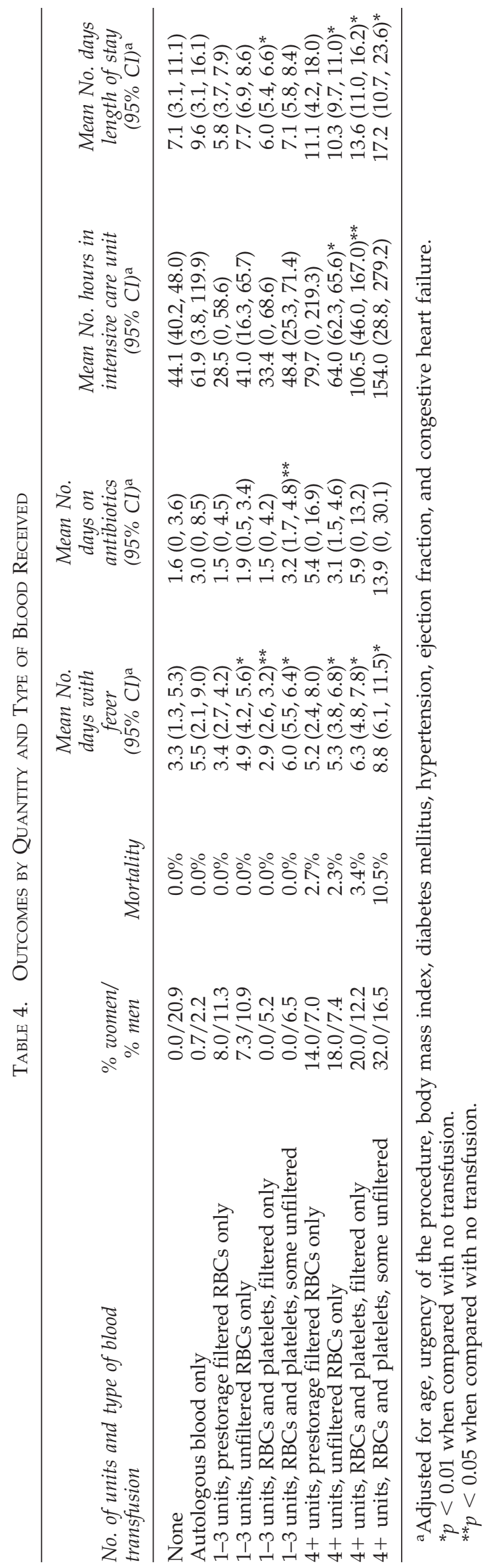


to reduce the number of days with fever for those who received one to three units. There was an increase (from 3.4 to 4.9 days) in the mean number of days with fever for patients who received one to three units of unfiltered vs filtered RBCs ( $p<$ $0.001)$. Likewise, there was an increase (2.9 to 6.0 days) in the mean number of days with fever for those who received one to three units of unfiltered vs. filtered RBCs and platelets $(p<0.001)$.

There was considerable variation in the number of days on antibiotics. Although the mean number of days on antibiotics ranged from 3.1 to 13.9 in patients who received four or more units of various types of blood components, none were significantly different from the mean for patients without a transfusion (mean of 1.6 days). The exception was patients who received one to three units of unfiltered RBCs and platelets, whose mean number of days on antibiotics was $3.2(p=$ 0.031).

The mean number of hours spent in an intensive care unit tended to increase with an increasing number of units of blood transfused. Patients who were transfused with four or more units of either unfiltered RBCs only (64 hours, $p<0.001$ ) or filtered RBCs and platelets (106.5 hours, $p=$ 0.043 ) spent a greater number of hours in intensive care than did patients without a transfusion (44.1 hours). Although the mean number of hours in the intensive care unit was 154 for those receiving four or more units of unfiltered RBCs and platelets, the difference was of marginal significance $(p=0.085)$ when compared with patients with no transfusion.

Hospital length of stay was greater in patients who received four or more units of either unfiltered RBCs only (10.3 days, $p<0.001)$, filtered RBCs and platelets (13.6 days, $p<0.001$ ), or unfiltered RBCs and platelets (17.2 days, $p=0.002$ ) compared with patients without a transfusion (7.1 days).

\section{DISCUSSION}

In this study, women experienced greater infection and mortality after cardiac surgery compared with their male counterparts. This may be due, at least in part, to the type and quantity of blood transfusion received. Women were more likely than men to receive an allogeneic blood transfusion as well as greater quantities of blood, even after consideration of preoperative hemat- ocrit, age, BMI, and clinical presentation. Allogeneic RBC and platelet transfusions were strongly associated with an increased risk of infection, pulmonary dysfunction, and death, particularly when nonleukoreduced blood was administered.

The relation between gender and transfusion has been reported and, similar to what we found here, remained after consideration of hematocrit levels. ${ }^{14}$ In a systematic review, female gender, preoperative anemia, advancing age, and small body size were found to be the most common predictors of perioperative allogeneic RBC transfusion. ${ }^{16}$

The risk of a poor outcome was greatest in those who received four or more units of either nonleukoreduced RBCs or who received any platelet transfusions. In a nationwide study of patients in intensive care units, a greater number of RBCs transfusions has been shown to be an independent predictor of longer stay in the intensive care unit and increased length of hospital stay, as well as increased mortality. ${ }^{17}$ Thirty-day mortality was 4-fold greater in patients who received more than four units of RBCs compared with patients without a transfusion. Furthermore, the use of restrictive criteria for transfusion, and thus reduced use of allogeneic blood, was shown to be just as effective as liberal criteria in critically ill and surgical patients, although the current evidence in cardiac patients is not yet sufficient. ${ }^{18-20}$

In a large population-based study in Canada prior to the implementation of universal leukoreduction of allogeneic blood, an increased risk of mortality in women was evident only within 30 days after the CABG procedure but not in the subsequent 10 years. ${ }^{21}$ Therefore, it is possible that the risk of infection from allogeneic blood may be short term; if so, it is during this period that vigilance about infection control would be most appropriate. Given that approximately $41 \%-71 \%$ of all Americans have a blood transfusion within their lifetimes, the effect of this exposure and the time at-risk for infection needs additional study. ${ }^{22}$

An association between transfusion and lung injury has been reported, and a rare consequence, transfusion-related acute lung injury, carries a $5 \%-10 \%$ risk of mortality. ${ }^{23}$ In a study of patients in four intensive care units at the Mayo Clinic, 7\% of those who received transfusions developed respiratory failure within 6 hours of the transfusion. ${ }^{24}$ Although mechanisms underlying these 
relationships were not a part of this investigation, proinflammatory agents in stored blood components, particularly platelets, have been implicated. ${ }^{25}$ For example, soluble CD40 ligand, a proinflammatory protein that accumulates during storage, has been shown to activate polymorphonuclear leukocytes in patients and may cause pulmonary endothelial damage and transfusion-related acute lung injury. ${ }^{26}$ Allogeneic blood has been shown to alter cytokine levels, increase humoral immunity, and decrease cell-mediated immunity. ${ }^{27}$ Immunological effects of allogeneic blood have been recognized since the 1970s and, since then, have been used to enhance graft survival in patients with solid organ transplants, particularly before the widespread use of immunosuppressant medications. ${ }^{4,28}$

\section{Limitations}

We were unable to assess the independent effects of platelets, as they were almost exclusively administered with RBCs in this population. Similarly, we could not assess the effects of prestorage vs. poststorage filtration because the type of filtration was unique to the particular blood component; all filtering of RBCs was conducted prior to storage, whereas all filtering of platelets was conducted after storage. Our data did show, however, that filtered blood components tended to decrease the number of days with fever compared with unfiltered blood. In addition, the data suggest that larger quantities of unfiltered RBCs and platelets result in a longer hospital stay and higher mortality.

As this study was not a randomized, controlled trial, we were unable to determine if the relation between transfusion and infection was due to confounding by indication; that is, the underlying reasons for prescribing a transfusion, and not the transfusion itself, may lead to detrimental outcomes. Fergusson et al. ${ }^{29}$ conducted a metaanalysis of the randomized, controlled trials in cardiac surgical patients who received leukoreduced vs. nonleukoreduced blood transfusion. They found an overall reduction in postoperative infection for patients receiving leukoreduced blood. Bilgin et al. ${ }^{30}$ also found a significant reduction in the infection rate in patients who received leukocyte-depleted erythrocytes (compared with buffy coat-depleted erythrocytes) in a randomized controlled trial of patients who underwent cardiac valve surgery. These studies, be- cause of their randomized design, provide evidence that confounding by clinical indication is not likely to explain improved patient outcomes with leukoreduced transfusion.

\section{CONCLUSIONS}

Immunomodulation from the receipt of nonleukoreduced allogeneic blood may increase the risk of infection, pulmonary dysfunction, and mortality during hospitalization for cardiac surgery. This is of particular significance for women, who are more likely to receive a greater quantity of blood than men. Measures should be taken to encourage research in alternatives to nonleukoreduced allogeneic blood and to increase infection control procedures during the period of immunomodulation. Wider use of leukoreduced RBC transfusions and multimodal approaches to blood conservation and anemia prevention, as in bloodless medicine and surgery programs, may provide particular benefit to women. ${ }^{31}$

\section{REFERENCES}

1. Jacobs AK. Women, ischemic heart disease, revascularization, and the gender gap. I Am Coll Cardiol 2006;47:63S.

2. Merrill C, Elixhauser A. Procedures in U.S. hospitals, 2003. Rockville, MD: Agency for Healthcare Research and Quality. HCUP fact book No. 7. AHRQ publication No. 06-0039. ISBN 1-58763-185-7, 2005.

3. Stover EP, Siegel LC, Parks R, et al. Variability in transfusion practice for coronary artery bypass surgery persists despite national consensus guidelines: A 24-institution study. Anesthesiology 1998;88: 327.

4. Opelz G, Terasaki PI. Improvement of kidney-graft survival with increased numbers of blood transfusions. N Engl J Med 1978;299:799.

5. Blumberg N, Heal JM. Transfusion and recipient immune function. Arch Pathol Lab Med 1989;113:246.

6. Blumberg N, Triulzi DJ, Heal JM. Transfusion-induced immunomodulation and its clinical consequences. Transfusion Med Rev 1990;4(Suppl 1):24.

7. Murphy PJ, Connery C, Hicks GL Jr, Blumberg N. Homologous blood transfusion as a risk factor for postoperative infection after coronary artery bypass graft operations. IThoracic Cardiovasc Surg 1992;104:1092.

8. Hill GE, Frawley WH, Griffith KE, Forestner JE, Minei JP. Allogeneic blood transfusion increases the risk of postoperative bacterial infection: A meta-analysis. I Trauma 2003;54:908. 
9. Triulzi DJ, Blumberg N, Heal JM. Association of transfusion with postoperative bacterial infection. Crit Rev Clin Lab Sci 1990;28:95.

10. Taylor RW, Manganaro L, O'Brien J, Trottier SJ, Parkar N, Veremakis C. Impact of allogenic packed red blood cell transfusion on nosocomial infection rates in the critically ill patient. Crit Care Med 2002;30:2249.

11. Leal-Novel SR, Rincón-Ferrari MD, Garcia-Curiel A, et al. Transfusion of blood components and postoperative infection in patients undergoing cardiac surgery. Chest 2001;119:1461.

12. Koch CG, Liang L, Duncan AI. Morbidity and mortality risk associated with red blood cell and bloodcomponent transfusion in isolated coronary artery bypass grafting. Crit Care Med 2006;34:1608.

13. Dunne JR, Riddle MS, Danko J, Hayden R, Petersen $\mathrm{K}$. Blood transfusion is associated with infection and increased resource utilization in combat casualties. Am Surgeon 2006;72:619.

14. Scott BH, Seifert FC, Glass PS, et al. Blood use in patients undergoing coronary artery bypass surgery: Impact of cardiopulmonary bypass pump, hematocrit, gender, age, and body weight. Anesth Analg 2003;97:958.

15. Rogers MAM, Blumberg N, Saint SK, Kim C, Nallamothu BK, Langa KM. Allogeneic blood transfusions explain increased mortality in women after coronary artery bypass graft surgery. Am Heart J 2006;152:1028.

16. Khanna MP, Hebert PC, Fergusson DA. Review of the clinical practice literature on patient characteristics associated with perioperative allogeneic red blood cell transfusion. Transfus Med Rev 2003;17:110.

17. Corwin HL, Gettinger A, Pearl RG, et al. The CRIT Study: Anemia and blood transfusion in the critically ill-Current clinical practice in the United States. $\underline{\text { Crit }}$ Care Med 2004;32:39.

18. Carson JL, Hill S, Carless P, Hébert P, Henry D. Transfusion triggers: A systematic review of the literature. Transfus Med Rev 2002;16:187.

19. Hébert PC, Wells G, Blajchman MA, et al. A multicenter, randomized, controlled clinical trial of transfusion requirements in critical care. $\mathrm{N}$ Engl J Med 1999;340:409.

20. Hébert PC, Yetisir E, Martin C, et al. Is a low transfusion threshold safe in critically ill patients with cardiovascular diseases? Crit Care Med 2001;29:227.

21. Guru V, Fremes SE, Tu JV. Time-related mortality for women after coronary artery bypass graft surgery: A population-based study. I Thorac Cardiovasc Surg 2004;127:1158.

22. Hay SN, Scanga L, Brecher ME. Life, death, and the risk of transfusion: a university hospital experience. Transfusion 2006;46:1491.

23. Swanson K, Dwyre DM, Krochmal J, Raife TJ. Transfusion-related acute lung injury (TRALI): Clinical and pathophysiologic considerations. Lung 2006;184:177.

24. Rana R, Fernandez-Perez ER, Khan SA, et al. Transfusion-related acute lung injury and pulmonary edema in critically ill patients: A retrospective study. Transfusion 2006;46:1478.

25. Spiess BD. Transfusion of blood products affects outcome in cardiac surgery. Semin Cardiothorac Vasc Anesth 2004;8:267.

26. Khan SY, Kelher MR, Heal JM, et al. Soluble CD40 ligand accumulates in stored blood components, primes neutrophils through CD40, and is a potential cofactor in the development of transfusion-related acute lung injury. Blood 2006;108:2455.

27. Twomley KM, Rao SV, Becker RC. Proinflammatory, immunomodulating, and prothrombotic properties of anemia and red blood cell transfusions. J Thromb Thrombolysis 2006;21:167.

28. Siemionow M, Agaoglu G. Role of blood transfusion in transplantation: A review. J Reconstructive Microsurg 2005;21:555.

29. Fergusson D, Khanna MP, Tinmouth A, Hébert PC. Transfusion of leukoreduced red blood cells may decrease postoperative infections: Two meta-analyses of randomized controlled trials. Can J Anesth 2004; 51:417.

30. Bilgin YM, van de Watering LM, Eijsman L, et al. Double-blind, randomized controlled trial on the effect of leukocyte-depleted erythrocyte transfusions in cardiac valve surgery. Circulation 2004;109:2755.

31. Goodnough LT, Shander A, Spence R. Bloodless medicine: Clinical care without allogeneic blood transfusion. Transfusion 2003;43:668.

Address reprint requests to: Mary A.M. Rogers, Ph.D. Department of Internal Medicine University of Michigan 300 North Ingalls, Suite 7E07 Ann Arbor, MI 48109-0429

E-mail: maryroge@umich.edu 
This article has been cited by:

1. James Rawn. 2008. The silent risks of blood transfusion. Current Opinion in Anaesthesiology 21:5, 664-668. [CrossRef]

2. Michael R. Savona, Samuel M. Silver. 2008. Erythropoietin-Stimulating Agents in Oncology. The Cancer Journal 14:2, 75-84. [CrossRef] 